\title{
A New Approach for PAPR Reduction of OFDM Signal Based on Signal Scrambling \#
}

\author{
Mohamed A. Abbas ${ }^{1}$, Safa Gasser ${ }^{2}$, Ashraf M. Aziz ${ }^{3}$ and Mohamed E. Khedr ${ }^{4}$
}

Accepted $15^{\text {th }}$ August 2014

\begin{abstract}
Orthogonal Frequency Division Multiplexing (OFDM) is an attractive and promising technique for fourth generation wireless communication. In general, the High Peak-to-Average Power Ratio (PAPR) of transmitted signals for OFDM systems reduces the system efficiency and hence increases the cost of the Radio Frequency (RF) power amplifier. In this paper, we propose a distortion-less PAPR reduction schemes for multi-carrier system. The new schemes combine both the Partial Transmit Sequence (PTS) and the Selected Mapping (SLM) techniques. The performance of the new schemes is measured using the Complementary Cumulative Distribution Function (CCDF) of the PAPR. The simulation results show that the two new combined schemes have a better PAPR performances compared to PTS and SLM. On the other hand, the price paid is a slight increase in the system complexity, as well as the side information transmitted.
\end{abstract}

Keywords: Orthogonal Frequency Division Multiplexing (OFDM); Peak-to-Average Power Ratio (PAPR); Concatenate Parallel Selected Mapping (C-PSLM); Concatenate Hybrid Parallel Selected Mapping (C-HPSLM); Complementary Cumulative Distribution Function (CCDF)

\section{Introduction}

Wireless communication systems evolved rapidly within the past three decades starting from the first generation in the 1980s. It depended mainly on analog signals and reached the fourth generation wireless systems with very high transmission rate. Thus, recent and future generation of wireless communication systems are characterized by a variety of applications ranging from low rate voice data to very high rate real-time streaming video data. OFDM is the latest multi-carrier modulation technique adopted by the current mobile wireless communications network.

OFDM is a very attractive technique for high-speed data transmission and has many advantages such as robustness in frequency selective fading channels, high spectral efficiency, immunity to inter-symbol interference and capability of handling very strong multipath [1]. OFDM has many disadvantages, one of which is the high PAPR of the transmitted signal. OFDM signal consists of a number of independently modulated sub-carriers, which causes a large instantaneous peak power compared to average power when added coherently. This large peak brings some problems in the transmitter like an increased complexity of RF power amplifier and efficiency reduction [2].

The PAPR is defined by Eq. (1); where $\mathrm{P}_{\text {peak }}$ represents the peak power in one OFDM symbol, and $\mathrm{P}_{\text {averge }}$ represents average power in the same OFDM symbol, E $\left[\left|\mathrm{x}_{\mathrm{n}}\right|^{2}\right]$ denotes the expected

$\overline{1,2 \& 4}$ Department of Electronics \& Communications Engineering Arab Academy for Science, Technology and Maritime Transport, Egypt Email:m.atef.abbas@aast.edu,safagasser@aast.eduandkhedr@aast.edu

${ }^{3}$ Department of Electrical Engineering, Al-Baha University, Saudi Arabia Email: amaziz64@ieee.org

\# This paper has been presented at the International Conference on Advanced Technology\&Sciences (ICAT'14) held in Antalya (Turkey), August 12-15, 2014. value of $x_{n}, x_{n}$ represents a discrete-time OFDM signal with $N$ sub-carriers after IFFT operation, and $\mathrm{x}_{\mathrm{n}}$ is expressed as in Eq. (2); where $\mathrm{N}$ is the number of sub-carriers.

$$
\begin{aligned}
& \text { PAPR }=\frac{P_{\text {peak }}}{P_{\text {averge }}}=\frac{\max \left\{\left|x_{n}\right|^{2}\right\}}{E\left[\left|x_{n}\right|^{2}\right]} \\
& x_{n}=\frac{1}{\sqrt{N}} \sum_{k=0}^{N-1} X_{k} \cdot e^{\frac{2 \pi n k j}{N}}
\end{aligned}
$$

In the literature the PAPR problem was tackled by three different methods: The first method is based on decreasing the number of sub-carriers. This affects the data rate, thus makes the OFDM loses its main advantages.

The second method is based on improving the RF amplifier, which avoids driving the amplified signal to saturation. It also prevents spectral growth of the multicarrier signal in the form of inter-modulation among sub-carriers and out-of-band radiation.

The third method is based on other reduction techniques [2],[3],[4]. These reduction techniques are divided into two broad categories. First, signal distortion, which reduce the PAPR by distorting the transmitted OFDM signal. Second, signal scrambling which scramble the transmitted OFDM signal using different methods to obtain low PAPR. The most popular methods used in signal distortion are three;

\subsection{Amplitude clipping and filtering $[5,6]$,}

This technique was proposed by O'Neill and Lopes in 1995 [5]. O'Neill and Lopes technique is considered the simplest technique for PAPR, as it only requires clipping the transmitted signal such that the peak amplitude becomes limited to a desired level; however there are two basic problems associated with this technique; the first problem is the introduction of selfinterference that degrades the Bit Error Rate (BER), while the second problem is the introduction of the nonlinear distortion for 
the OFDM signal. The nonlinear distortion introduced increases both the in and out of band radiation. Thus, filtering is suggested after clipping to reduce the out of band radiation. However it cannot reduce in-band distortion. On the other hand, clipping may cause some peak regrowth so that the signal after clipping and filtering will exceed the clipping level at some points. In order to overcome the problem of peak regrowth, a repeated clipping-andfiltering operation is suggested at the cost of an increased computational complexity.

2. Peak windowing was introduced by Pauli and Kuchenbeeker in 1998 [7]. The windowing technique tends to improve the clipping technique by introducing a suitable narrow-band window. The narrow-band window is used for the purpose of attenuating the peak of the transmitted signal. In addition, it reduces the out-ofband radiation.

3.Companding technique was introduced by H. Xiao, L. Jianhua, Z. Junli, J. Chuang, and G. Jun in 2001 [8]. This method is considered one of the most attractive schemes, due to fact that it gives the best system performance; that is high PAPR reduction at a low BER, with low system complexity without any loss in the date rate.

On the other hand, the most known proposed methods for signal scrambling are four;

1. Selected Mapping (SLM) was introduced by Bäuml, Fischer and Huber in 1996 [9]. This technique is based on the idea of creating different versions of the same signal by multiplying the original OFDM signal by different phased rotation. These signals are then fed through a selection phase, which selects the signal with the least PAPR.

2. Partial Transmit Sequence (PTS) [10] was introduced by Bäuml, Fischer and Huber in 1997. This technique is based on the idea of partitioning the original OFDM sequence into sub-blocks. The IFFT for each one of these sub-blocks is computed separately and then weighed by a phase factor. The phase factors are selected in such a way as to minimize the PAPR of the combined signal of all the sub-blocks.

3. Tone Reservation (TR) technique was introduced by Tellado and Cioffithe in 1998 [11], [12]. This method is an efficient technique to reduce the PAPR of a multi-carrier signal and is based on adding a dependent time domain signal to the original multi-carrier signal to reduce its peaks.

4. A combined PTS-SLM scheme was introduced by Wang, LanXun, Kang Yang, and Bin Xu in 2009 [15]. The combined technique improves the PAPR, as well as it reduces the overall system computational complexity.

In this paper we propose two new methods for PAPR reduction based on signal scrambling. The first method is based on combining two stages of SLM, while the second method is based on combining the first method with PTS together to obtain better PAPR reduction. Thus this paper is divided as follows: The SLM and PTS approaches of PAPR reduction are briefly described in Section II. The proposed method is explained in section III. The simulations results are introduced in Section IV and, finally the conclusion is given in Section V.

\section{The PAPR scheme using PTS and SLM}

In this section we will review two of the most important techniques in signal scrambling for PAPR reduction, namely SLM and PTS.

\subsection{PAPR using SLM scheme}

The SLM approach uses multiple-signal representation to reduce PAPR. SLM is based on the idea of creating different versions of the same signal by multiplying the original signal by different phased rotation. These signals are then fed through a selection phase, which selects the signal with the least PAPR [13].

A block diagram of SLM is shown in Fig.1. In SLM, the data source is portioned into $\mathrm{N}$ data blocks where $\mathrm{N}$ represents the number of sub-carriers in the OFDM system. Replicas of the same-portioned $\mathrm{N}$ data block are then multiplied by $\mathrm{V}$ statistically independent phase rotation sequences. The length of the phase sequence is the same length of the $\mathrm{N}$ data block. The output of the multiplier process is then fed through an IFFT algorithm followed by a PAPR calculation module. The phase rotated symbol sequence with the lowest PAPR is selected and transmitted with added side information [10]. We can also decrease the PAPR by increasing the $\mathrm{V}$ phase rotated symbol sequences.

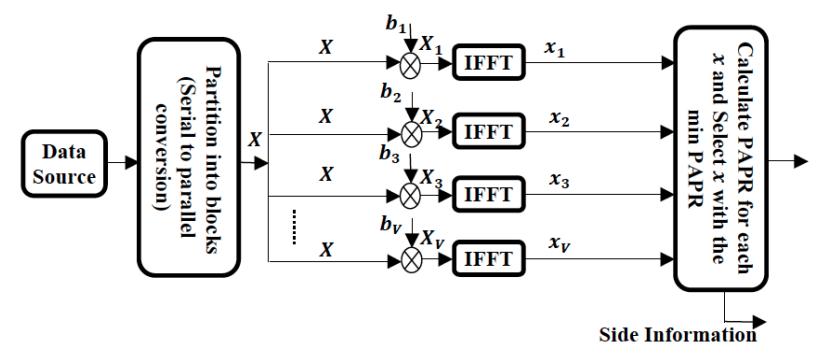

Fig. 1: A block diagram of SLM [Src: Bäuml, Fischer and Huber]

\subsection{PAPR using PTS scheme}

The basic idea of PTS algorithm is to divide the original OFDM signal into several sub-sequences, and for each sub-sequence, multiply by different weights until an optimum PAPR value is reached [14].

In PTS the percentage of PAPR reduction depends on the number of sub-blocks $M$ and the number of allowed phase factors $b$. In addition, sub-block partitioning may affect the PAPR reduction performance. There are three different methods of sub-block partitioning: adjacent, interleaved, and pseudo-random partitioning [2], [14].

A block diagram of PTS is shown in Fig.2 In PTS, the data source is portioned into $\mathrm{N}$ data blocks where $\mathrm{N}$ represents the number of sub-carriers in the OFDM system. The data block of length $\mathrm{N}$ is divided into $\mathrm{M}$ disjoints sub-blocks using adjacent partitioning. The disjoint sub-blocks consist of an adjacent set of sub-carriers and are all of equal size. These dis-joint sub-blocks are then fed to an IFFT algorithm. The output of the IFFT algorithm is then summed after multiplying them with a distinct rotating factor $b_{m}\left\{\right.$ where $b_{m}$ are weighing factors and $m=1$, $2 . . \mathrm{M}\}$.

Finally, the combination of the M disjoint sub-blocks is optimized in order to get the lowest PAPR. The previous process is then repeated again for each block. The main disadvantage of the PTS algorithm is that it requires the transmission of phase factors as side information, which results in data rate reduction.

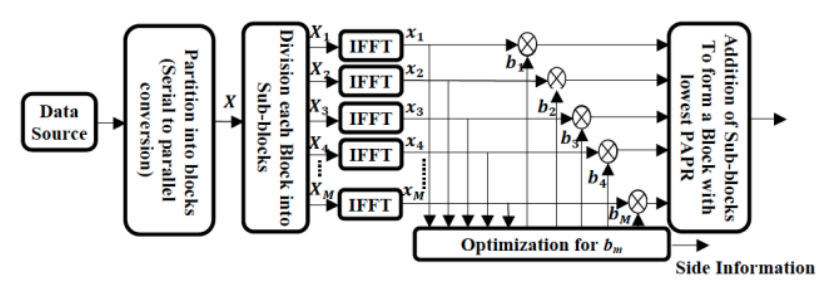

Fig. 2: A block diagram of PTS [Src: Bäuml, Fischer and Huber] 


\section{The Proposed algorithms}

In this section we give a description of the proposed algorithms. The proposed algorithms are hybrid techniques based on both SLM and PTS. The first algorithm is obtained by combining two stages of SLM in parallel, the results of which are concatenated to form the output sequence. This algorithm is referred to as Concatenate-Parallel SLM (C-PSLM). The second algorithm is similar to the first one, except that each SLM stage is followed by an FFT algorithm stage as well as PTS prior to the concatenation stage. The details of both proposed algorithms are given below.

\subsection{PAPR using C-PSLM scheme}

The C-PSLM algorithm is based on the idea of partitioning the input data block into two equal sub-blocks. Each of the subblocks is then fed through a regular SLM algorithm, taking into account that both SLM algorithms are identical. The results of both SLM algorithms are concatenated to form an output sequence of length $\mathrm{N}$. The whole idea of breaking the data is to get the best PAPR for both sequences then by concatenation we get a better overall reduction, as compared to a single SLM stage. Fig.3 illustrates a block diagram for the proposed C-PSLM algorithm.

It is worth mentioning that the C-PSLM algorithm requires more side information as compared to a single stage SLM. This excess in side information results in slight reduction of the data rate for the overall system.

\subsection{PAPR using C-HPSLM scheme}

In this proposed algorithm we combine the C-PSLM and PTS methods as shown in Fig.4. First, the C-PSLM approach is used but without the concatenation of the output signals. Each output signal of the two parallel stages are then passed through FFT algorithm, and then passed through a PTS block. Finally, the outputs of the two PTS blocks are concatenated. The phase factors and the rotation phase must then be transmitted as side information. The idea behind combining these two algorithms is to obtain signal with lower PAPR than in that obtained by $\mathrm{C}$ PSLM, SLM and PTS.

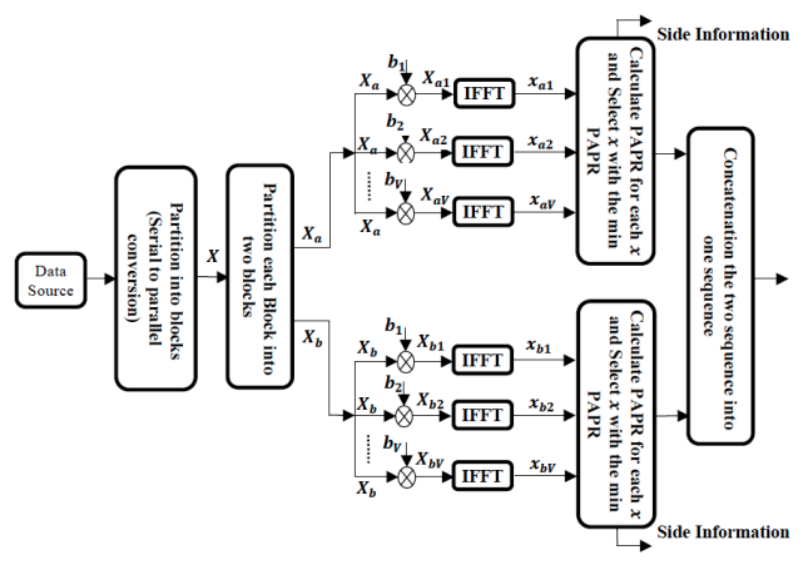

Fig. 3: A block diagram of C-PSLM

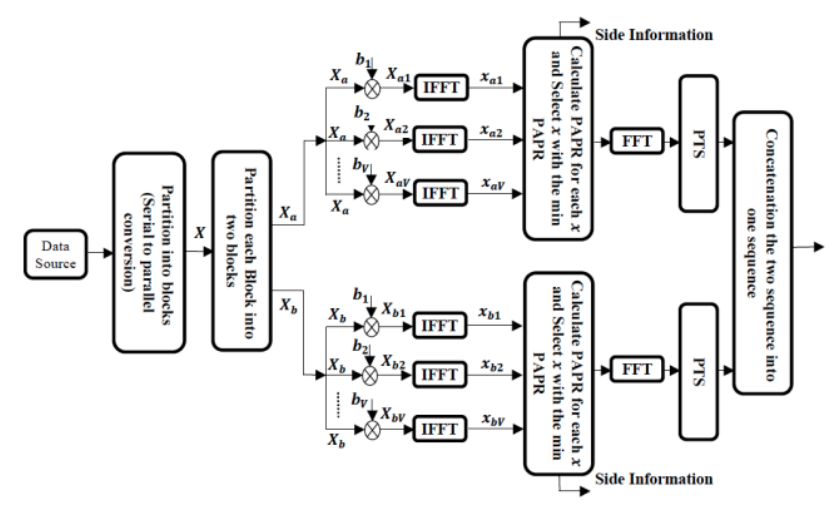

Fig. 4: A block diagram of C-HPSLM

\section{Simulation results}

The performance of a PAPR reduction scheme is usually measured by many factors: the complementary cumulative distribution function (CCDF), the BER, the PAPR reduction capability, the power increase in transmit signal, the loss in data rate, and the computational complexity increase. The CCDF of the PAPR is the most preferred measure for PAPR reduction techniques [2].

A MATLAB (R2010b) code was written to evaluate the performance of the proposed algorithms. CCDF curve was picked as a performance measure for PAPR reduction. A CCDF curve was plotted for each of the proposed algorithms, which plots the probability versus the PAPR in $\mathrm{dB}$.

\subsection{Simulation results of C-PSLM for different values of $\mathbf{V}$}

First, the performance of the C-PSLM with different values of $\mathrm{V}$ is compared. The simulation parameters considered are illustrated in Table 1 It is clear from Fig. 5 that by increasing the value of V, the probability of high PAPR decreases, as compared to the original OFDM signal. If the probability is held fixed at the value of $0.1 \%$, comparing the CCDF curves with different values of $\mathrm{V}$, it is observed that the PAPR value for $\mathrm{V}=2$ case is about $1 \mathrm{~dB}$ smaller than the original OFDM signal. Under the same condition, the PAPR value for $\mathrm{V}=8$ case is about $3 \mathrm{~dB}$ smaller than the original OFDM signal. However, comparing the $V=8$ and $\mathrm{V}=16$ cases, one can conclude that the performance difference between them is $0.25 \mathrm{~dB}$. From the curves obtained we deduce that linear growth of PAPR reduction performance cannot be achieved as we increase the value of $\mathrm{V}$.

Table 1. Simulation Parameter for C-PSLM

\begin{tabular}{|c|c|}
\hline Simulation Parameter & Value \\
\hline Number of sub-carriers (N) & 512 \\
\hline Number of sub-blocks V in SLM (stage a \& b) & $2,4,8,16,32$ and 64 \\
\hline Modulation scheme & QPSK \\
\hline Number of Symbols & 100,000 \\
\hline
\end{tabular}




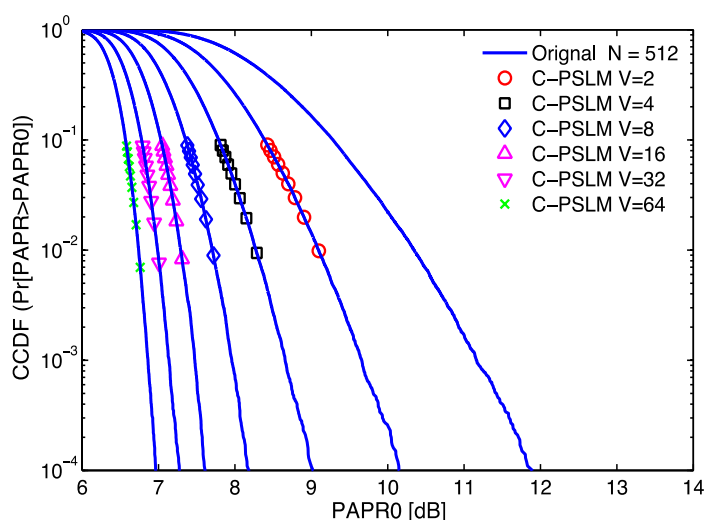

Fig. 5: A comparison of CCDF curves for C-PSLM

4.2. Comparison of C-HPSLM for different values of $V$ with fixed $M$

Second, the performance of the C-HPSLM with different values of $\mathrm{V}$ is compared for fixed value of $\mathrm{M}$. The simulation parameters considered are illustrated in Table 2. Adjacent partitioning was the method applied in simulating PTS. It is clear from Fig.6 that by increasing the value of $\mathrm{V}$, the probability of high PAPR decreases, as compared to the original OFDM signal. If the probability is held fixed at the value of $0.1 \%$ and $\mathrm{M}=4$, comparing the CCDF curves with different values of $\mathrm{V}$, it is observed that the PAPR value for $\mathrm{V}=2$ case is about $2.5 \mathrm{~dB}$ smaller than the original OFDM signal. Under the same condition, the PAPR value for $\mathrm{V}=8$ case is about $3 \mathrm{~dB}$ smaller than the original OFDM signal. However, comparing the $\mathrm{V}=8$ and $\mathrm{V}=16$ cases, one can conclude that the performance difference between them is $0.25 \mathrm{~dB}$. From the curves obtained, we deduce that linear growth of PAPR reduction performance cannot be achieved as we increase the value of $\mathrm{V}$ while fixing the value of M.

Table 2. Simulation Parameter for C-HPSLM

\begin{tabular}{|l|c|}
\hline \multicolumn{1}{|c|}{ Simulation Parameter } & Value \\
\hline Number of sub-carriers (N) & 512 \\
\hline Number of sub-blocks V in SLM (stage a\& b) & $2,4,8,16,32$ and 64 \\
\hline Number of disjoint sub-block M in PTS & 4 \\
\hline Modulation scheme & QPSK \\
\hline Number of Symbols & 100,000 \\
\hline
\end{tabular}

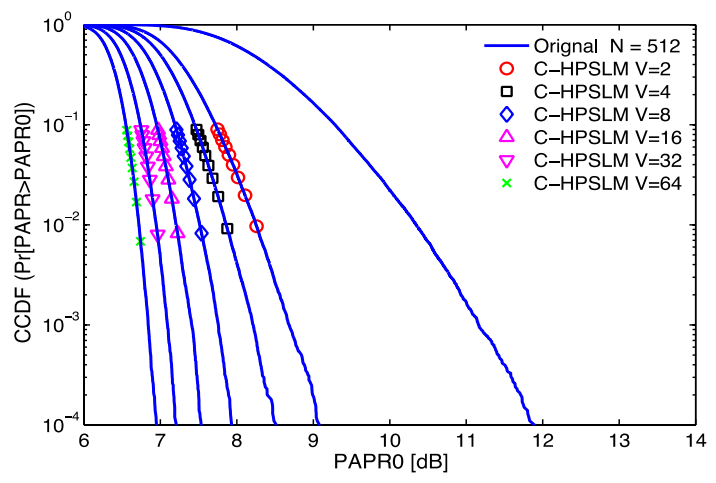

Fig. 6: A comparison of CCDF curves for C-HPSLM

4.3. Comparison between the two proposed algorithms and SLM and PTS
Third, the performance of the two proposed algorithms with SLM and PTS is given. The simulation parameters considered are illustrated in Table 3 From Fig.7 it is shown that the C-HPSLM gives higher PAPR reduction when compared to the C-PSLM, PTS and SLM techniques. Table 4 summarizes the percentage of reduction obtained from C-HPSLM, C-PSLM, PTS and SLM methods.

Table 3. Simulation Parameter for C-HPSLM, C-PSLM, SLM and PTS

\begin{tabular}{|l|c|}
\hline \multicolumn{1}{|c|}{ Simulation Parameter } & Value \\
\hline Number of sub-carriers (N) & 512 \\
\hline Number of sub-blocks V in SLM (stage a \& b) & 4 \\
\hline Number of sub-blocks V in SLM & 4 \\
\hline Number of disjoint sub-block M in PTS & 4 \\
\hline Modulation scheme & QPSK \\
\hline Number of Symbols & 100,000 \\
\hline
\end{tabular}

Table 4. Comparison for C-HPSLM, C-PSLM, SLM and PTS

\begin{tabular}{|c|c|c|c|}
\hline & $\begin{array}{c}\text { PAPR (dB) } \\
\text { at 0.001\% }\end{array}$ & $\begin{array}{c}\text { PAPR }_{\text {orignal }}(\mathbf{d B}) \\
- \text { PAPR }_{(\mathbf{d B})}\end{array}$ & $\begin{array}{c}\text { Percentage of } \\
\text { Reduction }\end{array}$ \\
\hline Orignal Signal & 11.89 & 0 & 0 \\
\hline SLM & 9.203 & 2.687 & $22.6 \%$ \\
\hline PTS & 10 & 1.89 & $15.9 \%$ \\
\hline C-PSLM & 9.024 & 2.866 & $24.1 \%$ \\
\hline C-HPSLM & 8.518 & 3.372 & $28.4 \%$ \\
\hline
\end{tabular}

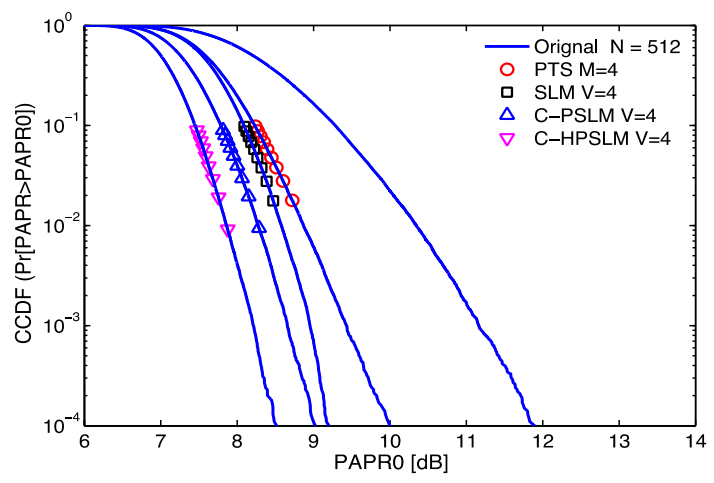

Fig. 7: A comparison of CCDF curves for C-HPSLM, C-PSLM, SLM and PTS

\section{Simulation results}

In this paper we proposed two new algorithms for PAPR reduction; namely C-PSLM and C-HPSLM. As shown in the paper, the C-PSLM and C-HPSLM are based on merging both classical SLM and PTS.

The simulation results showed that the two new algorithms outperform the classical PTS and SLM. In addition, the new algorithms are simple and easy to understand at the expense of increased system complexity and reduced data rate. Thus, future work is recommended to investigate ways to reduce the system complexity, as well as to reduce the amount of side information transmitted. 


\section{References}

[1] R. Van Nee and R. Prasad, "OFDM for Wireless Multimedia Communications", Artech House, Boston, Mass, USA, 2000.

[2] Y. Rahmatallah and S. Mohan, "Peak-to-average power ratio reduction in OFDM systems: A Survey and Taxonomy," IEEE Comm. Surveys \& Tutorials, vol. 15, no. 4, pp. 1567-1592, Fourth Quarter 2013.

[3] S. Han and J. Lee, "An Overview of Peak-To-Average Power Ratio Reduction Techniques for Multicarrier Transmission," IEEE Wireless Communications, vol. 12, no. 2, pp. 56-65, Apr. 2005.

[4] T. Jiang and Y. Wu, "An Overview: Peak to-Average Power Ratio Reduction Techniques for OFDM Signals," Broadcasting, IEEE Transactions on, vo1. 54, no. 2, pp. 257-268, June 2008.

[5] R. O'Neill and L.B. Lopes, "Envelope Variations and Spectral Splatter in Clipped Multicarrier Signals," IEEE conference proceedings PIMRC'95, Toronto, Ont., pp. 7176, 27-29 Sep. 1995.

[6] X. Li and L. J. Cimini, "Effect of Clipping and Filtering on the Performance of OFDM," IEEE Comm. Lett. , Vol. 2, no. 5, pp. 131-133, May 1998.

[7] M. Pauli and H.P. Kuchenbecker, "On the reduction of the out of band radiation of OFDM signals," IEEE conference proceedings ICC 98, Atlanta, GA, Vol. 3, pp. 1304-1308, 711 Jun. 1998.

[8] H. Xiao, L. Jianhua, Z. Junli, J. Chuang, and G. Jun, "Reduction of peak-to-average power ratio of OFDM signals with companding transform," IEE Electronic Letters, vol. 37, no. 8, pp. 506-507, April 2001.

[9] R. W. Bäuml, R. F. H. Fisher, and J. B. Huber, "Reducing the Peak-to-Average Power Ratio of Multicarrier Modulation by Selected Mapping," Elect. Lett., vol. 32, no. 22, pp. 2056-2057, Oct. 1996.

[10] S. H. Müller and J. B. Huber, "A Novel Peak Power Reduction Scheme for OFDM," Proc. IEEE PIMRC '97, Helsinki, Finland, pp. 1090-1094, 1-4 Sept. 1997.

[11] J. Tellado, Peak to Average Power Reduction for Multicarrier Modulation, Ph.D. dissertation, 2000: Stanford University.

[12] J. Tellado and J. M. Cioffi, "Efficient algorithms for reducing PAR in multicarrier systems," Proc. IEEE Int. Symp. Information Theory, Cambridge, MA, pp.191, 16-21 Aug. 1998.

[13] S. H. Müller and J. B. Huber, "OFDM with Reduced Peakto-Average Power Ratio by Optimum Combination of Partial Transmit Sequences," Elect. Lett., vol. 33, no. 5, pp. 368-369, Feb. 1997.

[14] L. J. Cimini, Jr. and N. R. Sollenberger, "Peak-to-Aver-age Power Ratio Reduction of an OFDM Signal Using Partial Transmit Sequences," IEEE Communications Letter, vol.4, no. 3, pp. 86-88, March 2000.

[15] L. Xun, Wang, K. Yang, and B. Xu. "Using the union algorithm of SLM and PTS to reduce PAPR in OFDM system," 2009 ISECS International Colloquium on Computing, Communication, Control, and Management. Vol. 4, Sanya, China, pp. 511-513, 8-9 Aug. 2009. 